\title{
Balancing equity and efficiency in the Dutch basic benefits package using the principle of proportional shortfall
}

\author{
E. J. van de Wetering $\cdot$ E. A. Stolk • \\ N. J. A. van Exel • W. B. F. Brouwer
}

Received: 4 October 2010/ Accepted: 5 August 2011/Published online: 26 August 2011

(C) The Author(s) 2011. This article is published with open access at Springerlink.com

\begin{abstract}
Economic evaluations are increasingly used to inform decisions regarding the allocation of scarce health care resources. To systematically incorporate societal preferences into these evaluations, quality-adjusted life year gains could be weighted according to some equity principle, the most suitable of which is a matter of frequent debate. While many countries still struggle with equity concerns for priority setting in health care, the Netherlands has reached a broad consensus to use the concept of proportional shortfall. Our study evaluates the concept and its support in the Dutch health care context. We discuss arguments in the Netherlands for using proportional shortfall and difficulties in transitioning from principle to practice. In doing so, we address universal issues leading to a systematic consideration of equity concerns for priority setting in health care. The article thus has relevance to all countries struggling with the formalization of equity concerns for priority setting.
\end{abstract}

Keywords Proportional shortfall - Priority setting · Equity $\cdot$ Social value $\cdot$ Resource allocation

Jel Classification $\mathrm{I} 10 \cdot \mathrm{I} 18$

E. J. van de Wetering $(\bowtie) \cdot$ E. A. Stolk .

N. J. A. van Exel · W. B. F. Brouwer

Institute for Medical Technology Assessment and Institute

of Health Policy and Management, Erasmus University

Rotterdam, 3000 DR, Rotterdam, The Netherlands

e-mail: vandewetering@bmg.eur.nl

\section{Introduction}

Economic evaluations are increasingly used to inform decisions regarding the allocation of scarce health care resources. They generally take the form of cost-utility analysis, in which incremental costs per gained QALY (quality-adjusted life year) are evaluated against some threshold to ascertain the intervention's value for money. In this procedure, QALY gains are (implicitly) valued equally irrespective of, for instance, the beneficiary or disease. Such practice has been an issue of debate, however, because accumulating evidence shows that the public may prefer some QALY gains over others (e.g., young over old) [1-3], often relating to a more equitable distribution of health and health care. Such notions of equity are normally not captured in economic evaluations where QALYs are typically weighted equally and often remain implicit in subsequent policy decisions, if included at all.

Almost three decades ago, researchers recognized that equity concerns could be incorporated into allocation decisions in the health care sector by weighting QALY gains according to some agreed upon equity principle [4], such as giving more weight to gains in the severely ill. Nonetheless, explicit QALY weighting is still uncommon. It seems that little has changed since Schwappach's [2] assertion that equity weighting, if considered at all, was at a developmental stage. However, given the growing pressure on health care budgets - partly due to (expensive) new technologies and increased demand stemming from demographic changes-we can expect that the process of allocating scarce health care resources will require increasing attention. Since decision makers increasingly use economic evaluations to inform their decisions [5], the discrepancies between recommendations based on economic evaluation outcomes and actual or publicly desired 
decisions may become more evident, as experienced in the UK, Netherlands, and Australia [6-9]. One explanation for such discrepancies is the presence of equity concerns that are insufficiently reflected in current economic evaluations [10]. Therefore, we need a more explicit and systematic incorporation of equity weights to obtain sustainable decisions.

Recent developments in the UK with respect to the funding of costly life-prolonging drugs illustrate the attention to equity concerns [11, 12]. In general, the National Institute for Health and Clinical Excellence (NICE) appears to adhere to the principle that 'a QALY is a QALY,' implying that all QALY gains should receive equal weight [13], but under that rule, it appears to have been difficult to come to sustainable decisions in the context of costly life-prolonging drugs. The appraisal committees thus now explicitly consider the "magnitude of the additional weight that would need to be assigned to the QALY benefits ... for the cost-effectiveness of the technology to fall within the current threshold range' [14]. This guideline, specific in terms of applicable interventions and open in terms of what weights might be considered appropriate, might be a first step in defining more general rules regarding equity weights.

In response to the equity problem, the Dutch have developed a decision-making framework that uses equity weights in defining the basic benefits package. Its primary criteria (proposed in 1991) are necessity, effectiveness, and efficiency [15]. The first refers to a notion of equity based on the need for medical intervention; the latter two refer to the merits of the intervention itself [16]. The three criteria inform the decision for including an intervention in the benefits package. Equity weights thus are intended to be an integral part of rather than exceptions to the rule. In this context, broad consensus appears to exist for using a particular operationalization of necessity as basis for equity weighting, i.e., the concept of proportional shortfall [1619]. Although choosing a specific equity principle may lead to a more systematic and transparent way of using equity weights to set priorities in the Dutch health care system, it also requires justification.

The Dutch and UK experiences suggest that including equity concerns in practice is not straightforward. Arriving at a systematic consideration raises two important questions: (i) Which equity principle(s) are used to base QALY weights on? (ii) How can we derive practically applicable QALY weights that are in line with the chosen principle(s)? These difficult and inherently normative questions complicate the formalization of equity concerns.

To our knowledge, the Netherlands is the first country where decision makers and health economists have been involved in a joint effort to formulate an equity principle and develop a model for putting it into practice. Although the concept of proportional shortfall is not yet firmly implemented, that does appear to be the ultimate goal.
Evaluating the proportional shortfall concept and the support for it in more detail is therefore directly relevant to the Dutch situation as well as other countries currently struggling with the formalization of equity concerns for priority setting in health care.

In this article, we will evaluate the proportional shortfall concept as used in the Dutch health care context, with a focus on the above-mentioned research questions on QALY weighting within the context of a chosen principle.

\section{Economic evaluation and equity}

Equity weights are integrated into economic evaluations to adequately consider costs and benefits. The common decision rule for economic evaluations is shown in Eq. 1 [20]:

$V_{\mathrm{QALY}_{i}} * \Delta \mathrm{QALY}_{i}-\Delta \cos$ ts $>0$,

where $V_{\mathrm{QALY}_{i}}$ denotes the monetary value society attaches to a QALY of type $i$ and the subscript $i$ is used to distinguish QALY gains according to some equity principle. $\triangle \mathrm{QALY}_{\mathrm{i}}$ represents the number of type $i$ QALYs gained; $\Delta$ costs are the associated costs. Both are derived relative to some relevant comparator. Accordingly, the term $V_{\mathrm{QALY}_{i}} * \Delta \mathrm{QALY}_{\mathrm{i}}$ reflects the benefits related to the intervention. Put simply, Eq. 1 indicates that incremental benefits of the intervention need to outweigh its incremental costs to be eligible for funding.

In common economic evaluations, however, the monetary value component in the benefits is not included in the equation. Rather the focus is only on $\triangle \mathrm{QALY}_{\mathrm{i}}$ (commonly without any distinction between QALYs, following 'a QALY is a QALY no matter who gets it') and $\Delta$ costs. This means that common economic evaluations in the field of health care do not directly address $V_{\mathrm{QALY}_{i}}$. As such, these analyses are basically partial economic evaluations. This is easily demonstrated by reordering Eq. 1:

$\frac{\Delta \text { cos ts }}{\Delta \text { QALY }_{i}}<V_{\text {QALY }_{i}}$

where the costs per QALY gained of type $i$ have to be lower than the societal value attached to that particular type of QALY in order to be eligible for funding. Only then a common cost-effectiveness or cost-utility analysis becomes a full economic evaluation as noted in Eq. 1. (It should be noted that irrespective of how QALYs, costs, and potentially the value of QALYs are derived, the equivalence between a common cost-benefit analysis and the decision rule based on a cost-utility analysis may still be questioned [21, 22]. Also note, that a fuller discussion on decision rules, under different assumptions regarding decision contexts (e.g. fixed budgets) and goals (e.g. focusing on health or the consumption value of health) is provided by Claxton et al. [23]. 
Commonly, one threshold is set for all QALYs, regardless of the context in which they are gained. However, in line with Eq. 2, different cost-effectiveness ratios may be acceptable for different types of QALYs gained if the value of a QALY is allowed to vary, for instance, on the basis of the disease or beneficiary characteristics. If so, rather than having one threshold value for all QALYs, we have a range whose endpoints are defined by the lowest and highest possible values attached to a gained QALY in a particular context. These different contexts may well refer to notions of equity. It should be noted that such a practice is equal to keeping the threshold value constant but attaching 'equity weights' to the QALY gains on the lefthand side of Eq. 2. This implicitly ensures the use of an appropriate threshold value since the equity weights may simply be regarded as the relative values of different QALYs. In the Dutch context, the former approach is taken, i.e., different threshold values are used when the burden of illness is high (e.g., acute life-threatening diseases) or low (e.g., toenail fungus). The latter approach appears to have been adopted for life-prolonging drugs in the UK, i.e., keeping the threshold fixed but weighting QALY gains.

To illustrate that one can either vary the threshold values or attach equity weights to QALYs, we use a hypothetical example in which the QALY value is allowed to vary between young and old. For children, the value is highest, say $1 \mathrm{QALY}=€ 100,000$. For people aged 90 and over, the value is lowest, say $1 \mathrm{QALY}=€ 5,000$. Let the reference value of a QALY be that of a 40-year-old, or $€ 40,000$. One way to judge a $\mathrm{CE}$ ratio of gains in children is to compare the $\mathrm{CE}$ ratio to the threshold line running from 5,000 to 100,000 , where for this intervention the high endpoint is relevant. Alternatively, 'equity weights' can be used to adjust the CE ratio itself, which can be judged against the common threshold of 40,000. In this example, the equity weight of QALYs for children relative to the 40-year-old reference group is $2.5\left(v_{\text {children }} / v_{40}\right.$ year olds $=100,000 /$ $40,000)$. Thus, in order to use equity weights appropriately, we (implicitly) compare the relative values of different QALY gains to some reference group with the standard threshold. Subsequently, such equity weights can be multiplied with the QALY gains in the CE ratio (i.e., $\Delta C /$ $2.5 * \Delta E$ ), which indirectly corrects the threshold value used.

The above demonstrates how using a flexible threshold is basically equal to attaching appropriate equity weights to different QALYs and comparing them to a single, relevant threshold. In that sense, the Dutch and UK approaches are similar, albeit the UK approach currently seems to be used in only a few specific circumstances. A crucial question, however, is which equity principle should guide the derivation or evaluation of equity weights (research question (i)). To this end, we next discuss the well-known principles of fair innings and prospective health, followed by discussion of the principle selected in the Dutch context, proportional shortfall.

\section{Fair innings and prospective health}

Which (combination of) equity concept(s) is most suitable for equity weighting? Several have been proposed even more equity concepts are imaginable [24-26]. A problem is that improved equality with one particular definition of equity may be (necessarily) accompanied by greater inequalities in the context of a different definition [27]. Selecting one (or more) principle(s) to guide the derivation or evaluation of equity weights is thus important and not straightforward. Two important principles are fair innings and prospective health. Fair innings, roughly speaking, strives for equity in lifetime health, while prospective health is more concerned with people's health expectations, regardless of experienced health. An aspect both principles share is that their basis is found within the health domain, i.e., they both focus on health characteristics of beneficiaries, not on aspects like gender or income. The principles differ, however, in that one strives to equalize lifetime health and the other prospective health.

The fair innings approach, advocated by Williams [24], is based on the assumption that everyone is entitled to some 'normal' span of life or health achievement. Anyone failing to achieve this has in some sense been disadvantaged in terms of lifetime health, while anyone getting more than this is living on 'borrowed time' [24]. This assumption implies that QALY gains in people who have had their fair innings should be valued lower than QALY gains in people who are expected to get less than their fair innings. Thus, the equity weights depend on the expected lifetime QALY total, therefore also considering past health losses and age is a key element (as proxy for lifetime health achievement), resulting in higher weights for QALY gains in relatively young persons and lower ones for those in relatively older persons.

In contrast, the principle of prospective health bases equity weights on the expected QALY profile of a person in the case of no treatment. This aligns with an alternative definition of need, namely, expected ill health over the remaining years of life [28]. Prospective health considers the expected health (including death) in future years in the case of non-intervention and distributes QALY gains initially to those with the worst prognosis if left untreated [28]. The approach appears to be related to the Rule of Rescue, which implies that rescuing identifiable individuals facing avoidable death should have priority over other types of care $[29,30]$. While prospective health 
Table 1 Illustrating fair innings and prospective health

\begin{tabular}{llll}
\hline Patient group & QALY consumed & $\begin{array}{l}\text { QALYs remaining } \\
\text { (prospective health) }\end{array}$ & $\begin{array}{l}\text { Expected QALY } \\
\text { total (fair innings) }\end{array}$ \\
\hline A (immediate death) & 60 & 0 & 60 \\
B (younger) & 40 & 1 & 41 \\
\hline
\end{tabular}

incorporates non-identifiable individuals and non-lifethreatening conditions, both prioritize people with poor health prospects.

The different perspectives of fair innings and prospective health obviously result in different equity weights. As seen in Table 1, group A faces immediate death and group $B$ has one remaining QALY. However, group B consists of younger persons who, consequently, have enjoyed fewer QALYs than persons in group A. Adhering to the equity principle of prospective health, group A would have priority because they face immediate death. The fair innings principle, on the other hand, would prioritize group B since it comprises younger people, or, put more precisely, it has a lower lifetime quality-adjusted life expectancy [28, 31, 32].

A number of empirical studies have found at least some public support for both the principles of fair innings and prospective health $[1,2,33]$, although it may depend on the context of the decision [34]. As mentioned, age is important to the fair innings principle. Both Dolan et al. [1] and Schwappach [2] have found in their reviews that the majority of studies reveal support for giving less weight to health gains in older people, but Schwappach argues that age preferences vary across countries, study designs, and context. Additionally, both reviews note that age weighting may reflect underlying rationales other than the fair innings principle. For example, people may prefer health gains in young people because they expect them to last longer. Separation of the different rationales places specific requirements on the design of studies, which are not always met. Furthermore, Shah's recent review [33] indicates that the public prefers to prioritize individuals in poorer health rather than those in better health without treatment, even if it results in lower overall health gains [1, 2]. Shah, however, [33] emphasized that the strength of the support should be estimated more precisely to gain a true reflection of it.

There appears to be little evidence showing that either equity principle reflects the distributional preferences of society completely or one fully lacks support. In that regard, we should note that people may in fact adhere to both principles: someone may feel that (holding other things constant, i.e., health prospects) young people should receive priority over older people and, at the same time, feel that (holding other things constant, i.e., age) people with worse health prospects should receive priority over those with better health prospects. In the Dutch context, therefore, the equity principle proposed was a measure of severity labelled 'proportional shortfall', which contained elements of both fair innings and prospective health [37]. We should note that the Dutch decision, while deliberatively taken and explicitly justified, should be seen as a first, pragmatic attempt to find an equity principle that is practically applicable and supported by the public.

\section{The principle of proportional shortfall}

The concept of proportional shortfall adopts the normative viewpoint that priority should be given to those patients who lose the greatest proportion of their remaining health expectancy due to some illness if the illness remains untreated. In other words, measurements of inequalities in health should concentrate on the fraction of QALYs lost due to illness, relative to remaining life expectancy. Proportional shortfall (PS) can be measured on a scale from 0 (no health loss) to 1 (complete loss of remaining health) using the following formula:

$\mathrm{PS}=\frac{\text { Disease related QALY loss }}{\text { Remaining QALY expectation in absence of the disease }}$,

where the denominator reflects the remaining QALY expectation in normal health, which could, for example, be determined on the basis of the age and gender. The numerator presents the QALY loss, which is determined by deducting a patient's QALY expectancy given the disease without treatment from the remaining QALY expectancy in absence of the disease. The proportional shortfall is 1 for all patients who face a threat of immediate death, irrespective of their age. Since they will lose $100 \%$ of their remaining life expectancy, they all receive equal weight. Likewise, if a young patient with a normal QALY expectation of 40 loses 20 QALYs, he or she will get the same equity weight as an older patient with a QALY expectation of 2 who stands to lose 1 QALY: both patients lose $50 \%$ of their remaining life expectancy. Since proportional shortfall is a relative measure, both younger and older individuals can experience a low or high proportional shortfall. For another example, a 30-year-old losing 1 of 40 remaining QALYs would receive low treatment priority (1/ $40=0.025$ ), while a 70 -year-old losing 1 of 5 remaining QALYs would receive higher priority $(1 / 5=0.2)$. 
Whereas fair innings and prospective health equalize absolute health outcomes in terms of total and future health, proportional shortfall proposes to equalize relative attainments. By doing so, the concept combines elements of both fair innings and prospective health [37]. In accordance with fair innings, proportional shortfall is concerned with disease-related QALY loss; at the same time, in accordance with prospective health, it takes the remaining QALY expectation without treatment into account [35]. Therefore, the principle may be perceived as an intermediate position between fair innings and prospective health.

Obviously, this does not necessarily mean that proportional shortfall is in any sense 'better' than the other two. Its use must be justified, both normatively and empirically. A limitation to normative justification is that unlike, say, fair innings, the concept is not derived from a particular theory about distributive justice. Instead, normative arguments for both prospective health and fair innings are assumed to be compelling; thus, given that both principles result in different prioritizations, a reconciliation of or trade-off between the two is required. While balancing two principles that appear to have some normative and empirical support may intuitively make sense, whether the resulting combination has the same (or even a better) moral status than the individual principles themselves remains questionable [36].

One convincing argument for this (and thus proportional shortfall) may be whether the latter reflects societal preferences better than either of its underlying principles. Evidence is thus far inconclusive, as is the only head-tohead comparison of all three concepts in which Stolk et al. [31] performed an experiment in a sample of Dutch health policy makers, researchers, and students to explore support for the individual principles. Observed rank orders of ten conditions were compared with rank orders based on the three equity principles. While both fair innings and proportional shortfall were highly correlated with the observed rank order, rank orderings based on prospective health showed only a moderate correlation coefficient with the respondents' ranking. Fair innings seemed to slightly outperform proportional shortfall, but neither offered a fully accurate description of societal preferences: There were always cases where the predicted rank ordering diverged from the observed. It was therefore concluded that more (elaborate) research was required before firm conclusions could be drawn about which concept best reflects societal preferences.

Despite the limited empirical and theoretical evidence to support proportional shortfall, broad consensus exists in the Netherlands to use it for equity weighting. The choice was partly pragmatic, subject to possible adjustment according to incoming evidence and ongoing experience. From the outset, it was obvious that the adoption of any equity principle would not be without problems. To avoid perfection becoming the enemy of the good, however, efforts were made to operationalize proportional shortfall for use in practice. We highlight this in the following section, while addressing the issue of how practically applicable QALY weights can be derived in line with the chosen principle and how they can subsequently be used in decision making on the allocation of scarce health care resources.

\section{The practice of proportional shortfall}

As indicated, three criteria have been particularly important in the delineation of the basic benefits package in the Dutch context: necessity, effectiveness, and efficiency. While proposed and well received about two decades ago [15], adopting them for practical use has proven difficult and controversial. Effectiveness, which has always been a dominant criterion in the health care sector, was least controversial but not efficiency, which is nonetheless increasingly used and accepted. It has been operationalized primarily through cost-effectiveness and cost-utility analyses, and most widely applied in the context of new pharmaceuticals. 'Necessity,' while intuitively important, eluded definition and measurement and thus remained barely applied as a (systematic) selection criterion in practice until 2002. Attempts to exclude services based on the criterion of necessity commonly provoked political or societal debate. Proportional shortfall was introduced to provide a more systematic and quantitative definition of necessity, which also solved its problem of dichotomy: interventions were deemed either necessary or unnecessary with no in-between. This proved virtually impossible as a proper definition of necessity, and a clear and universal cutoff point between unnecessary and necessary (or, for that matter, cost-effective and cost-ineffective) care was lacking. Mostly, there would be (groups of) patients for whom it would be difficult to label the intervention as unnecessary or cost-ineffective [37].

The introduction of proportional shortfall made it possible to quantify the necessity criterion and to integrate it with or relate it to results regarding (cost-)effectiveness in the decision-making framework. Basically, the threshold of cost per QALY (i.e., the value of the QALY) was allowed to vary with the necessity of the intervention, creating a decision framework in line with Eq. 2. The idea was that society is willing to pay more for an intervention, given the underlying proportional shortfall, considered more necessary [38]. Put differently, a less favorable cost-effectiveness ratio is acceptable for an intervention in the context of a greater proportional shortfall, i.e., when the treatment is deemed to be more necessary, and unacceptable when the 


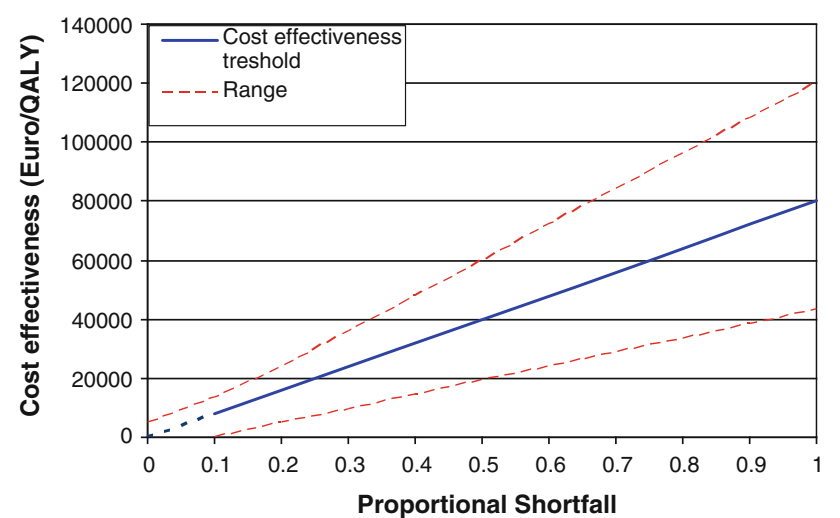

Fig. 1 Cost-effectiveness threshold varying with Proportional Shortfall

associated proportional shortfall is low. This decisionmaking framework is illustrated in Fig. 1.

While the framework is increasingly supported [17, 19], it is unclear how it has affected decisions and it is likely that it is currently used predominantly as a conceptual framework. One reason may be that it is relatively unclear how the threshold of costs per QALY gained varies with proportional shortfall. The equity weights placed on the different QALY gains (the value of gained QALYs relative to the underlying proportional shortfall) remain uncertain, although existing evidence may inform policy makers to some extent (see for example [41]). In that sense, the threshold and margins in Fig. 1 are largely tentative. Recently, a maximum threshold height of some $€ 80,000$ has been suggested [17, 39], although the choice lacks sound basis [40]. Furthermore, it has been suggested that social willingness to pay for QALY gains in the case of a very low burden of disease (proportional shortfall less than 10\%) might not exist at all in the context of a collectively funded health insurance scheme (which explains the dotted threshold line near the origin of the graph in Fig. 1). ${ }^{1}$ The shape of the curve would ultimately be based on adequately derived Dutch evidence regarding the relative valuations of QALY gains in different proportional shortfall contexts. A linear relationship has thus been proposed as a pragmatic starting point [18] but, even under this assumption, we need to be able to adequately judge the proportional shortfall in different circumstances to use the framework in practice.

The framework is thus currently more conceptual than prescriptive in assessing health care intervention. Supporting this may be the fact that the shortcomings of proportional shortfall have barely been discussed. As highlighted by the fair innings and prospective health

\footnotetext{
1 Asserting that a disease has a low necessity of treatment is in itself difficult. A relatively small health loss may be due to something severe during a small period of time or something relatively mild but chronic. Such profiles may be evaluated differently, as discussed later in the text.
}

principles, not all consequences of the proportional shortfall principle may be in line with common conceptions of an equitable distribution of health care. Perhaps the most counterintuitive implication of the principle of proportional shortfall is that anyone facing imminent death should receive the maximum necessity score of 1 , since all remaining health will be lost. This seems hard to defend when comparing between patients that differ substantially in age. Indeed, proportional shortfall assigns a necessity score of 1 when all remaining health is lost, regardless of the absolute number of life-years lost. The principle is indifferent to whether a 3 -year-old is losing 80 years or an 80-year-old is losing 3 years (when both expected to become 83 otherwise), but in practice, many people judge intervention to be more necessary in the former situation [40], making it conceivable that the result conflicts with society's equity principles. Likewise, since women have a higher life expectancy than men, an absolute QALY loss at a certain age will have more weight for men than women (for instance when comparing breast cancer and prostate cancer in certain age groups), and it is unclear whether this would be judged equitable [35, 42, 43].

Moreover, it became apparent that the operationalization of proportional shortfall required numerous normative choices that have important impacts on final outcomes. Coming to practically applicable proportional shortfall scores that are in line with public preferences is therefore not straightforward [35]. For instance, the calculation of proportional shortfall in preventive treatments requires clear normative choices. Considering that many who receive preventive treatment will never experience the negative health effects precluded by the intervention, which group is relevant? While the treated group may be relevant in a cost-utility analysis of a preventive intervention, it may not be when calculating the proportional shortfall of the underlying disease. Calculating proportional shortfall over the entire group would result in a very low average proportional shortfall since only a small percentage of the treated group would actually experience a health loss. This in turn results in low priority for (primary) preventive action, but the very aim of the intervention is to avoid health loss in those who would experience it without the preventive intervention. Then, it seems reasonable to calculate the proportional shortfall in the subgroup only, resulting in a higher proportional shortfall and threshold value. (The latter position is taken in the Netherlands.) Consequently, normative choices are necessary in defining the relevant group in which to determine proportional shortfall, which does not necessarily coincide with the population involved in the economic evaluation.

The timeframe for calculating proportional shortfall is another issue. Should the onset of preventive treatment be the starting point from which to calculate proportional shortfall? Or should it be the moment at which the negative health effects would have actually occurred? Obviously, 
the shorter timeframe will increase the proportional shortfall. Consider, for example, that a preventive intervention reduces a risk factor that, left untreated, results in death 20 years hence, reducing lifespan by 10 years. Until the moment of death, patients are without health loss. Calculating proportional shortfall starting from treatment time means that the first 20 years are in health and only the final 10 years are lost, resulting in a proportional shortfall of $33 \%$. Calculating from the moment of illness, the proportional shortfall is $100 \%$ (since then 10 of the remaining 10 life-years are lost). In the Netherlands, it has been argued that since society is likely to feel quite different about acute death than about a predicted (or certain) death in 20 years, calculating proportional shortfall from the moment of treatment would be more appropriate. Again, this is a normative choice, with substantial influence on results.

An additional problem exists in handling episodic diseases. An average proportional shortfall of 0.04 can result from a stable yet mild condition that causes a loss of 0.04 per day as well as from a disease that is primarily latent (no health loss for 350 days per year), but leaves the patient in agony during the episode (a loss of 1.0 during the remainder of the year). In the current operationalization of proportional shortfall, such episodes are simply averaged over the full year (as in normal QALY calculations). However, the appropriateness and justification of such a simple method of transforming health profiles into proportional shortfall scores can be disputed. Can we really conclude on such a basis that a certain illness has a modest severity? It appears that the variation over time may be important here too; yet, how could or should this be included in the calculation of proportional shortfall?

The above illustrates that not just the choice of an equity principle is normative; putting whatever it is into practice requires additional normative choices. Whatever the chosen principle, it appears inevitable that counterintuitive prioritizations may result in certain circumstances. Clearly, therefore, decision makers should be aware of additional and potentially conflicting equity considerations.

\section{Discussion}

Explicit inclusion of equity weights in the decision-making framework for allocation decisions in the health care sector has become increasingly important. A pragmatic start has been made in the Netherlands with the principle of proportional shortfall, which adopts the normative viewpoint that when people stand to lose relatively more of their remaining health, a higher cost per QALY threshold is appropriate. It thus quantifies the criterion of necessity in the Dutch decision-making framework. The higher the proportional shortfall, the more necessary the intervention.
This article highlights that the approach is not without problems. Both the normative basis and empirical support warrant further study. Of particular concern are situations where the consequences of proportional shortfall diverge from public preferences. For example, it seems hard to defend that avoiding a full loss of all remaining health would be equally important when the choice concerns either a very large or small absolute QALY loss, i.e., young and old people, respectively. Whether proportional shortfall adequately reflects societal preferences in such cases is uncertain and information on the circumstances of misalignment is pivotal in refining the principle and its employment.

We also highlighted that operationalizing proportional shortfall (or of any equity principle for that matter) involves normative choices that can have a profound effect on outcomes, like in the case of preventive interventions. It is crucial that these normative choices are as widely discussed as those embedded in cost-effectiveness analyses.

The highlighted shortcomings of proportional shortfall clearly should not be misinterpreted as a plea to replace it with a different equity concept such as fair innings. Indeed, whatever principle is chosen, similar shortcomings and normative choices will arise in transitioning from principle to practice. Since different notions of equity-all of which have some support in some instances-will always conflict in certain circumstances [27], conflicts with societal preferences will be inevitable. At this moment, there is no conclusive evidence that another equity principle reflects the distributional preferences of society better than proportional shortfall. It seems more appropriate, given the relatively strong (political) support for the equity concept of proportional shortfall in the Dutch context, to further test, develop, and refine the principle and its operationalization. For example, one might consider incorporating age weights in proportional shortfall in order to better reflect societal preferences. ${ }^{2}$ Obviously, this requires more and detailed research on relevant societal preferences as well as public debate. Improving the quantification of necessity will most likely be a lengthy and difficult process.

The current situation, while perhaps far from perfect, may be seen as an important and perhaps essential step in the development of an appropriate set of equity weights in the Netherlands. The associated quantification of necessity should improve consistency and transparency in the decision-making process. Meanwhile, experience from the systematic use of proportional shortfall will potentially improve the principle and its practical use.

Besides proportional shortfall or health profiles of beneficiaries in general, many other factors may be relevant for decision making in relation to equity considerations.

\footnotetext{
${ }^{2}$ Obviously, this also depends on whether one wishes to consider societal preferences to be a good guide for normative choices.
} 
Reviews by Dolan et al. [1] and Schwappach [2] have identified numerous factors besides health attainments or prospects that appear to influence the relative valuation of QALYs, such as prior health consumption, culpability, age, having dependents, and socioeconomic status. More recent studies have added to this field [33,44], but so far most involve small and unrepresentative samples, the studies are quite context specific, and findings are sometimes contradictory. It seems difficult at this stage to be conclusive regarding the relative weights given to these considerations in an empirical sense. While it may be interesting and helpful to analyze such additional equity concerns in relation to proportional shortfall, such empirical work should coincide with normative debates regarding whether such additional (or alternative) concerns should be included in the decision-making process. For instance, even if the public (on average) considers culpability important in fair allocation of health care resources, wanting to institutionalize such sentiments is questionable if only because Dutch legislation prohibits it.

Another challenge in the Dutch context is further quantification of the decision model. Currently, the QALY value is unclear, and how it varies with different proportional shortfall percentages and which equity weights should be placed on various QALY gains needs to be investigated further. To use the decision-making framework in practice, the (relative) values assigned to QALY gains for different levels of proportional shortfall have to be elicited, for instance, from the public. Different methods have been used such as willingness to pay, person tradeoff, or discrete choice analysis [3]. Which technique best captures the preferences of society may depend on the research question and whether relative weights of various equity concerns will be investigated simultaneously in combined trade-offs.

In conclusion, although proportional shortfall provides important information for decision makers by acceptably quantifying the necessity of treatment in the Dutch context, it clearly does not perfectly capture societal preferences. Sufficient room should be left in the decision-making framework and process to judge whether the equity weights accurately reflect the public preferences in particular circumstances and to improve on principles and practice if so indicated. More generally, the Dutch experience with equity considerations in relation to economic evaluations has given insight into the difficulties related to the choice for and operationalization of an equity concept for the allocation of scarce health care resources. Although the Dutch experiences are based on the concept of proportional shortfall, similar issues are likely to occur when opting for other equity concepts. Therefore, the Dutch experiences can provide helpful lessons for countries currently struggling with the important issue of formalization of equity concerns in priority setting in health care.
Acknowledgments This study was financially supported by Zorgonderzoek Nederland (ZonMW), Netherlands Organisation for Health Research and Development (project number 1520020011). We would like to thank Erik Nord for his useful comments on an earlier version of this paper. The usual disclaimer applies.

Open Access This article is distributed under the terms of the Creative Commons Attribution Noncommercial License which permits any noncommercial use, distribution, and reproduction in any medium, provided the original author(s) and source are credited.

\section{References}

1. Dolan, P., Shaw, R., Tsuchiya, A., Williams, A.: QALY maximisation and people's preferences: a methodological review of the literature. Health Econ. 14, 197-208 (2005)

2. Schwappach, D.L.B.: Resource allocation, social values and the QALY: a review of the debate and empirical evidence. Health Expect. 5(3), 210-222 (2002)

3. Cookson, R., Drummond, M., Weatherly, H.: Explicit incorporation of equity considerations into economic evaluation of public health interventions. Health Econ. Policy Law 4(02), 231-245 (2009)

4. Wagstaff, A.: QALYs and the equity-efficiency trade-off. J. Health Econ. 10(1), 21-41 (1991)

5. Schreyögg, J., Stargardt, T., Velasco-Garrido, M., Busse, R.: Defining the "Health benefit basket" in nine european countries. Eur. J. Health Econ. 6, 2-10 (2005)

6. Pronk, M.H., Bonsel, G.J.: Out-patient drug policy by clinical assessment rather than financial constraints? Eur. J. Health Econ. 5(3), 274-277 (2004)

7. Dakin, H.A., Devlin, N.J., Odeyemi, I.A.O.: "Yes", "No" or "Yes, but"? multinomial modelling of NICE decision-making. Health Policy 77(3), 352-367 (2006)

8. Devlin, N., Parkin, D.: Does NICE have a cost-effectiveness threshold and what other factors influence its decisions? A binary choice analysis. Health Econ. 13(5), 437-452 (2004)

9. George, B., Harris, A., Mitchell, A.: Cost-effectiveness analysis and the consistency of decision making: evidence from pharmaceutical reimbursement in australia (1991-1996). Pharmacoeconomics 19(11), 1103-1109 (2001)

10. Rutten, F., Van Busschbach, J.: How to define a basic package of health services for a tax funded or social insurance based health care system? Eur. J. Health Econ. 2(2), 45-46 (2001)

11. Cookson, R., McCabe, C., Tsuchiya, A.: Public healthcare resource allocation and the rule of rescue. Br. Med. J. 34(7), 540 (2008)

12. Appleby, J., Devlin, N., Parkin, D., Buxton, M., Chalkidou, K.: Searching for cost effectiveness thresholds in the NHS. Health Policy 91(3), 239-245 (2009)

13. Rawlins, M.D., Culyer, A.J.: National institute for clinical excellence and its value judgments. Br. Med. J. 329(7459), 224 (2004)

14. NICE: Appraising life-extending, end of life treatments (2009)

15. Dunning, A.J.: Kiezen en delen Advies in Hoofdzaken Van De Commissie Keuzen in De Zorg. Albani, Den Haag (1991)

16. CVZ: Breedte geneesmiddelenpakket. College voor Zorgverzekeringen, Amstelveen (2001)

17. RVZ: Zinnige en duurzame zorg. RVZ rapport, Den Haag (2006)

18. RVZ: Rechtvaardige en duurzame zorg. Den Haag (2007)

19. CVZ: Pakketbeheer in de praktijk. Diemen: CVZ Rapport (2006)

20. Gravelle, H., Brouwer, W., Niessen, L., Postma, M., Rutten, F.: Discounting in economic evaluations: stepping forward towards optimal decision rules. Health Econ. 16(3), 307-318 (2007) 
21. Bleichrodt, H., Quiggin, J.: Life-cycle preferences over consumption and health: when is cost-effectiveness analysis equivalent to cost-benefit analysis? J. Health Econ. 18(6), 681-708 (1999)

22. Dolan, P., Edlin, R.: Is it really possible to build a bridge between cost-benefit analysis and cost-effectiveness analysis? J. Health Econ. 21(5), 827-843 (2002)

23. Claxton, K., Paulden, M., Gravelle, H., Brouwer, W.B.F., Culyer, A.J.: Discounting and decision making in the economic evaluation of health care technologies. Health Econ. 20(1), 2-15 (2011)

24. Williams, A.: Intergenerational equity: an exploration of the 'fair innings' argument. Health Econ. 6(2), 117-132 (1997)

25. Williams, A., Cookson, R.: Equity in health. Handb. Health Econ. 1, 1863-1910 (2000)

26. Nord, E.: Concerns for the worse off: fair innings versus severity. Soc. Sci. Med. 60(2), 257-263 (2005)

27. Sen, A.: Inequality Reexamined. Oxford University Press, Oxford (1995)

28. Dolan, P., Olsen, J.A.: Equity in health: the importance of different health streams. J. Health Econ. 20(5), 823-834 (2001)

29. McKie, J., Richardson, J.: The rule of rescue. Soc. Sci. Med. 56(12), 2407-2419 (2003)

30. Hadorn, D.C.: Setting health care priorities in oregon. JAMA 265(17), 2218-2225 (1991)

31. Stolk, E.A., Pickee, S.J., Ament, A.H.J.A., Busschbach, J.J.V.: Equity in health care prioritisation: an empirical inquiry into social value. Health Policy 74(3), 343-355 (2005)

32. Nord, E.: Concerns for the worse off: fair innings versus severity. Soc. Sci. Med. 60(2), 257-263 (2005)
33. Shah, K.K.: Severity of illness and priority setting in healthcare: a review of the literature. Health Policy 93(2-3), 77-84 (2009)

34. Oliver, A.: A fair test of the fair innings? Med. Decis. Mak. 29(4), 491 (2009)

35. Stolk, E.A., van Donselaar, G., Brouwer, W.B.F., Busschbach, J.J.V.: Reconciliation of economic concerns and health policy: illustration of an equity adjustment procedure using proportional shortfall. Pharmacoeconomics 22(17), 1097 (2004)

36. Cookson, R., Dolan, P.: Principles of justice in health care rationing. J. Med. Ethics 26(5), 323 (2000)

37. Boer, B.: Onderzoek op maat: Een verkenning van factoren voor het gebruik van medical technology assessment (2002)

38. CVZ: Vervolgonderzoek breedte geneesmiddelenpakket. College voor Zorgverzekeringen, Amstelveen (2002)

39. Zwaap, J., Mastenbroek, C.G., van der Heiden, L.A.: Pakketbeheer in de praktijk 2. College voor Zorgverzekeringen, Amstelveen (2009)

40. Brouwer, W.: De basis van het pakket: Urgente uitdagingen voor de opzet en inzet van economische evaluaties in de zorg (2009)

41. Nord, E., Pinto, J.L., Richardson, J., Menzel, P., Ubel, P.: Incorporating societal concerns for fairness in numerical valuations of health programmes. Health Econ. 8(1): 25-39 (1999)

42. Stolk, E.A.: Uitwerking van het pakketprincipe noodzakelijkheid; dimensie ziektelast. College voor Zorgverzekeringen, Diemen (2009)

43. Johannesson, M.: Should we aggregate relative or absolute changes in QALYs? Health Econ. 10(7), 573-577 (2001)

44. Green, C., Gerard, K.: Exploring the social value of health-care interventions: a stated preference discrete choice experiment. Health Econ. 18(8), 951-976 (2008) 